\title{
Operative wound-related complications after cranial revascularization surgeries
}

\author{
${ }^{*}$ Keisuke Takanari, MD, PhD, ${ }^{1}$ Yoshio Araki, MD, PhD, ${ }^{2}$ Sho Okamoto, MD, PhD, ${ }^{2}$ \\ Hideyoshi Sato, MD, ${ }^{1}$ Shunjiro Yagi, MD, PhD, ${ }^{1}$ Kazuhiro Toriyama, MD, PhD, ${ }^{1}$ \\ Kinya Yokoyama, MD, ${ }^{2}$ Kenta Murotani, $\mathrm{PhD},{ }^{4}$ Shigeyuki Matsui, $\mathrm{PhD},{ }^{3}$ \\ Toshihiko Wakabayashi, MD, PhD, ${ }^{2}$ and Yuzuru Kamei, MD, $\mathrm{PhD}^{2}$
}

Departments of ${ }^{1}$ Plastic and Reconstructive Surgery, ${ }^{2}$ Neurosurgery, and ${ }^{3}$ Biostatics, Nagoya University Graduate School of Medicine; and ${ }^{4}$ Center for Advanced Medicine and Clinical Research, Nagoya University Hospital, Nagoya, Japan

OBJECT Intracranial revascularization surgeries are an effective treatment for moyamoya disease and other intracranial vascular obliterative diseases. However, in some cases, wound-related complications develop after surgery. Although the incidence of wound complication is supposed to be higher than that with a usual craniotomy, this complication has rarely been the focus of studies in the literature that report the outcomes of revascularization surgeries. Here, the relationship between intracranial revascularization surgeries and their complications is statistically assessed.

METHODS Between October 2004 and February 2010, 71 patients were treated using cerebral revascularization surgeries on 98 sides of the head. The relationship between wound complications and operative technique was retrospectively assessed. Multivariate logistic regression analysis was performed to identify the risk factors of wound complication, including operative technique, age, sex, diabetes mellitus (DM), hypertension, hyperlipidemia, and smoking history.

RESULTS In total, there were 21 (21.4\%) operative wound complications. Of these 21 complications, there were 14 $(66.7 \%)$ minor complications and 7 (33.3\%) major complications. No statistically significant relationship was found between wound complications and any surgical procedure. A trend toward severer complications was demonstrated for the procedures that used both STA branches ("double" procedures) in comparison with the procedures that used only 1 STA branch ("single" procedures, $p=0.016$, Cochran-Armitage trend test). Multivariate logistic regression analysis also revealed that double procedures demonstrated a significantly higher incidence of wound complications than single procedures (OR 3.087, $p=0.048$ ). DM was found to be a risk factor for wound complication (OR 9.42, $p=0.02)$, but age, sex, hypertension, and hyperlipidemia were not associated with the incidence of complications. Even though the blood supply to the scalp is abundant due to 5 arteriovenous systems, sometimes cutaneous necrosis develops after intracranial revascularization surgeries. The galeal blood supply is thought to be crucial for preventing wound-related complications. Special care is also thought to be required for DM patients.

CONCLUSIONS Revascularization surgeries seemed to demonstrate a higher risk of wound-related complications. Double-type procedures, which use both branches of the STA, and a history of DM were found to be risk factors for wound-related complications. Attention should be paid to the design of the galeal incision and vessel harvest line. Also, special attention should be paid to patients with DM.

http://thejns.org/doi/abs/10.3171/2014.12.JNS132602

KEY WORDS cranial revascularization surgery; extracranial to intracranial bypass; infection; skin necrosis; superficial temporal artery-to-middle cerebral artery anastomosis; wound-related complication; vascular disorders

\footnotetext{
ABBREVIATIONS BA = basilar artery; DM = diabetes mellitus; EDAMS = encephalo-duro-arterio-myo-synangiosis; EDAS = encephalo-duro-arterio-synangiosis; EMS = encephalo-myo-synangiosis; ICA = internal carotid artery; MCA = middle cerebral artery; MMD = moyamoya disease; SCA = superior cerebral artery; SPECT = singlephoton emission computed tomography; STA = superficial temporal artery; STSG = split-thickness skin graft.

SUBMITTED January 13, 2014. ACCEPTED December 5, 2014.

INCLUDE WHEN CITING Published online July 3, 2015; DOI: 10.3171/2014.12.JNS132602.

DISCLOSURE The authors report no conflict of interest concerning the materials or methods used in this study or the findings specified in this paper.

* Drs. Takanari and Araki contributed equally to this work.
} 
$\mathrm{O}$ PERATIVE wound-related complications are rare after craniotomy surgery without harvesting scalp vessels. It is known that the vascular network in the scalp is well developed with several arteriovenous systems, which meet the demands of tissue growth and differentiation in the scalp. ${ }^{23}$ Layers of tissues, such as the skin, subcutaneous tissue, galea, and pericranium, support and protect the cranial bone. Despite such rich vascularity, skin necrosis sometimes develops at the surgical site and is difficult to salvage. Even worse, skin necrosis can be a life-threatening problem when infection arises in the nonvascularized bone used in the primary operation. ${ }^{7}$

Intracranial revascularization surgeries are an efficient treatment for moyamoya disease (MMD) - which is characterized by progressive stenosis or occlusion of the bilateral internal carotid arteries (ICAs) that is associated with a compensatory abnormal vascular network ${ }^{4,8,11,12,14,21}$ and cerebral major artery occlusive diseases such as arterial occlusion and aneurysm. The surgical procedure involves several surgical techniques, including superficial temporal artery-to-middle cerebral artery (STA-MCA) bypass, superficial temporal artery-to-superior cerebral artery (STA-SCA) bypass, encephalo-duro-arterio-synangiosis (EDAS), and encephalo-duro-arterio-myo-synangiosis (EDAMS). These revascularization procedures have a specific common feature: switching the blood supply from the scalp to the brain by harvesting the STA. Although this switching is obviously necessary for facilitating cerebral blood flow, it compromises the blood supply to the scalp. For this reason, operative wound-related complications are considered to have a higher incidence in cerebral revascularization surgeries that use the STA than regular craniotomies. However, these complications have rarely been the focus of past studies that analyzed the outcome of these procedures.

In this study, we retrospectively reviewed the outcomes of cerebral revascularization surgeries performed at our institute and focused on the possible risk factors of wound complications.

\section{Methods}

Seventy-one patients underwent cerebral revascularization surgeries on 98 sides of the head between October 2004 and February 2010. All information was retrospectively collected and included in the study. A summary of the patients is shown in Table 1 . Our study population included 69 cases of MMD, 9 cases of pseudo-MMD, 7 ICA aneurysms, 6 ICA occlusions, 2 MCA aneurysms, 3 MCA occlusions, and 2 basilar artery (BA) occlusions. There were 36 male and 35 female patients, and the mean age was 32.5 years (SD 20.7 years; range $1-75$ years).

Information about the patient characteristics, operative procedures, and postoperative courses were retrospectively collected. The patient characteristics included age, sex, history of diabetes, hypertension, hyperlipidemia, and smoking history. Primary surgery was performed by a neurosurgeon (S.O. or Y.A.). The "single"-type operative procedures included STA-MCA bypass, STA-SCA bypass, EDAS, and EDAMS, in which one of the STA branches was used. The "double"-type procedure used both STA branches and con-
TABLE 1. Summary of surgical indications

\begin{tabular}{cc}
\hline Primary Disease & No. of Operative Sides \\
\hline MMD & \\
\hline MMD $(n=78)$ & 69 \\
\hline Pseudo-MMD & 9 \\
\hline Non-MMD $(n=20)$ & 7 \\
\hline ICA aneurysm & 6 \\
\hline ICA occlusion & 2 \\
\hline MCA aneurysm & 3 \\
\hline MCA occlusion & 2 \\
\hline BA occlusion & 98 \\
\hline Total &
\end{tabular}

sisted of a combination of single-type procedures. Patients who received high-flow bypass (arterial graft), parental artery occlusion, or encephalo-myo-synangiosis (EMS) but did not undergo the procedure with STA harvesting were excluded from this study. Double-type procedures for direct or indirect revascularization were performed to resolve severely hemodynamically compromised patients who had frequent ischemic events and poor cerebral circulation. Single-type procedures were performed in other patients. Information about the postoperative course included time of occurrence, severity of wound complications, and how these complications were managed. All wounds were primarily assessed by a neurosurgeon, and patients were referred to a plastic surgeon when needed. Wound complications were classified as minor or major by examination by 2 or more plastic surgeons. Wound complication with congestion, which results in superficial necrosis in the epidermal level or the loss of smooth adaptation, was classified as a minor complication and treated with ointment or simple resuturing. Major complications included cases that had full-thickness skin necrosis and consequently skin defects. These complications required tissue transfer, such as a local flap and/or skin graft. Osteomyelitis was also categorized as a major complication.

\section{Statistical Analysis}

One-way ANOVA was used to compare multiple samples. The Cochran-Armitage trend test was used to assess the trends of the surgical procedures toward wound complication. Multivariable analysis using multivariable logistic regression analysis was performed to determine the risk factors for wound complication, and the odds ratio for each risk factor was calculated. All statistical analyses were performed using SAS Analytics pro 9.3 (SAS Institute). Results with $\mathrm{p}<0.05$ were considered significant.

\section{Results}

A summary of the procedures and operative wound complications is shown in Table 2. In total, there were 21 (21.4\%) operative wound complications. Of these $21 \mathrm{com}-$ plications, there were $14(66.7 \%)$ minor complications and 7 (33.3\%) major complications.

No statistically significant differences were observed 
TABLE 2. Summary of surgical procedures and wound-related complications

\begin{tabular}{lrrrr}
\hline & No. of & \multicolumn{3}{c}{ Wound Complications } \\
\cline { 3 - 5 } \multicolumn{1}{c}{ Operative Procedure* } & Procedures & None & Minor & Major \\
\hline Single Type & & & & \\
\hline$\quad$ STA-MCA bypass & 75 & 48 & 6 & 1 \\
\hline EDAMS & 2 & 0 & 2 & 0 \\
\hline EDAS & 1 & 0 & 1 & 0 \\
\hline STA-SCA bypass & & & & \\
\hline Double Type & 21 & 12 & 5 & 4 \\
\hline STA-MCA bypass $(\times 2)$ & 2 & 1 & 0 & 1 \\
\hline STA-MCA bypass + EDAMS & 7 & 7 & 0 & 0 \\
\hline STA-MCA bypass + EDAS & 2 & 2 & 0 & 0 \\
\hline EDAMS $(\times 2)$ & 1 & 1 & 0 & 0 \\
\hline EDAS $(\times 2)$ & 98 & 77 & 14 & 7 \\
\hline Total & &
\end{tabular}

* Single type indicates procedures that only used 1 branch of the STA; double type indicates procedures that used 2 branches of the STA.

between operative wound complications and the surgical procedures (data not shown). However, a trend toward severer complications was demonstrated by the procedures that used both STA branches (double procedures) in comparison with the procedures that used only 1 STA branch (single procedures, $\mathrm{p}=0.016$, Cochran-Armitage trend test, Table 3 ). This trend was confirmed by the multivariable logistic regression analysis, in which double procedures demonstrated a significantly higher incidence of developing wound complications than single procedures (OR 3.087, $\mathrm{p}=0.048$, Table 4). Diabetes was also found to be a risk factor for wound complication (OR 9.42, $\mathrm{p}=$ 0.02). Other factors such as age, sex, hypertension, hyperlipidemia, and smoking did not demonstrate a significant correlation to the incidence of wound complication.

Improved cerebral blood flow was identified using single-photon emission computed tomography (SPECT) in all cases. All patients with operative wound complications were successfully treated and achieved complete wound healing.

\section{Illustrative Cases}

\section{Case 1}

A 67-year-old woman underwent left external carotid artery-to-MCA bypass and left STA to MCA bypass (STA-MCA bypass) due to a left ICA aneurysm. A $3 \times 8-$ $\mathrm{cm}$ skin congestion was observed along the incision line 3 days after surgery, and necrosis developed over 7 days postoperation (Fig. 1A). Reconstructive surgery was performed 22 days after primary surgery. The necrotic tissue, including the skin and subcutaneous tissue, was debrided. Because the underlying galeal tissue was not available due to necrosis at this level, reconstructive surgery was performed using a local flap and meshed split-thickness skin graft (STSG). A $7 \times 10-\mathrm{cm}$ sized local flap was designed in the area behind the defect, and the flap was dissected under the galeal layer. Then, the flap was transposed to the
TABLE 3. Summary of anastomosis types and wound-related complications*

\begin{tabular}{ccccc}
\hline & & \multicolumn{3}{c}{ Wound Complications } \\
\cline { 3 - 5 } Anastomosis Type & No. & None & Minor & Major \\
\hline Single & 65 & 55 & $9(13.8 \%)$ & $2(3.1 \%)$ \\
\hline Double & 33 & 22 & $5(14.7 \%)$ & $5(15.6 \%)$ \\
\hline Total & 98 & 77 & 14 & 7 \\
\hline
\end{tabular}

* There was a statistically significant difference in the incidence of wound complications between single- and double-type anastomoses $(p=0.016)$.

defect, and the donor site was covered by meshed STSG obtained from the thigh (Fig. 1B). No complications were observed after reconstructive surgery during follow-up, and the wound completely healed.

\section{Case 2}

A 48-year-old man underwent right STA-MCA bypass and EMS due to MMD. Osteomyelitis developed 3 months after surgery, and the infected bone was removed and an artificial ceramic bone was implanted. However, a second infection developed, and the patient was referred to the plastic surgery department (Fig. 2A and B). Reconstructive surgery using a free-vascularized omental flap and titanium mesh was performed 15 months after primary surgery. The artificial bone and infected tissue were removed, and then the wound was irrigated with saline. The right facial artery and right common facial vein were dissected and prepared as recipient vessels. The free omental flap was elevated with the right gastroepiploic artery and vein, and it was prepared for transfer. The omental flap was then placed on the exposed dura, and a titanium mesh plate was positioned on the omental flap and fixed to the adjacent healthy bone. The mesh was enclosed by folding the remaining omental flap over the superficial surface of the plate (Fig. 3A). The meshed STSG was then placed on the omental flap, and the aim was not to compress the vascular pedicle (Fig. 3B). No complications were seen during follow-up after surgery, and the wound completely healed (Fig. 4A and B).

\section{Discussion}

The arterial vascular distribution to the scalp is supplied by 5 pairs of major arteries. ${ }^{1,5,13,22,23,26}$ Three pairs-

TABLE 4. Multivariate analysis of risk factors for wound-related complications

\begin{tabular}{lccc}
\hline \multicolumn{1}{c}{ Variable } & OR & $95 \% \mathrm{Cl}$ & $\mathrm{p} \mathrm{Value}$ \\
\hline Double-type procedure & 3.09 & $1.01-9.45$ & 0.048 \\
\hline Female sex & 1.14 & $0.36-3.57$ & 0.822 \\
\hline Age & 0.98 & $0.94-1.02$ & 0.363 \\
\hline DM & 9.42 & $1.42-62.3$ & 0.020 \\
\hline Hypertension & 4.26 & $0.76-24.0$ & 0.100 \\
\hline Hyperlipidemia & 3.53 & $0.64-19.7$ & 0.150 \\
\hline Current smoker & 1.01 & $0.20-5.03$ & 0.994 \\
\hline
\end{tabular}




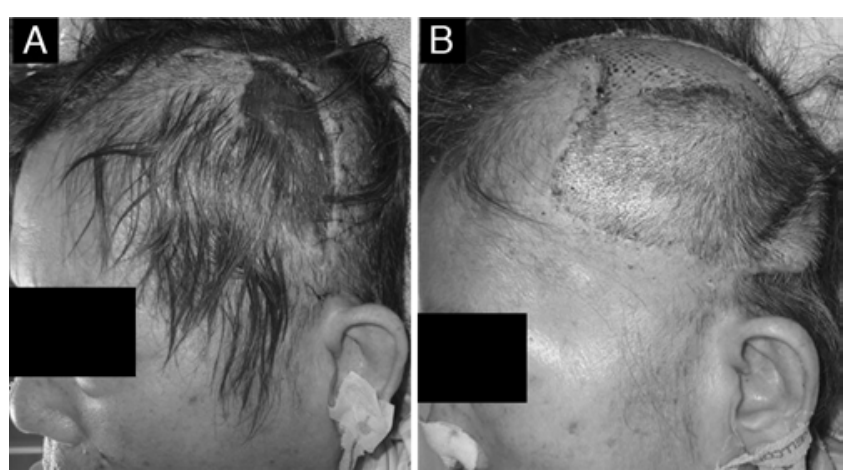

FIG. 1. Case 1. A: Preoperative photograph of the wound. B: Photograph obtained 1 month after reconstructive surgery.

the STA, occipital, and retroauricular arteries-arise from the external carotid artery system and cover the temporal, parietal, frontal (superficial temporal), and occipital (occipital and retroauricular) scalp areas. The remaining 2 pairs - the supraorbital artery and supratrochlear artery (which are the terminal branches of the ophthalmic artery that arises from the ICA system)-distribute blood to the frontal and parietal scalp areas. These 5 arteries supply the cutaneous, subcutaneous, and galeal layers while they anastomose each other. Among these arteries, the STA has the largest territory. Among these layers, the galeal layer contains the largest amount of blood in the scalp since it contains the major arteries. The small perforating vessels that branch out from the galea contribute to the scalp's blood supply. ${ }^{23}$ The venous vascular distribution, along with these major arteries, basically drains into the internal or external jugular veins. Despite such rich vascularity, skin necrosis sometimes develops after head operations. Even worse, when infection arises in the nonvascularized bone, it may affect the blood flow of the anastomosed vessels or result in osteomyelitis in the bone flap.

Wound-related complications are rare after craniotomy surgery without harvesting scalp vessels. Some studies, which discuss complications after craniotomy surgery without harvesting scalp vessels, suggest that wound infection occurs in about $5 \%$ of all patients $(1 \%-11 \%),{ }^{6,7,10,20,25} \mathrm{In}$ the report by Katsuta et al., ${ }^{10} 17.6 \%$ of patients who underwent cerebral revascularization surgery developed cutaneous necrosis without the appearance of infection. Based
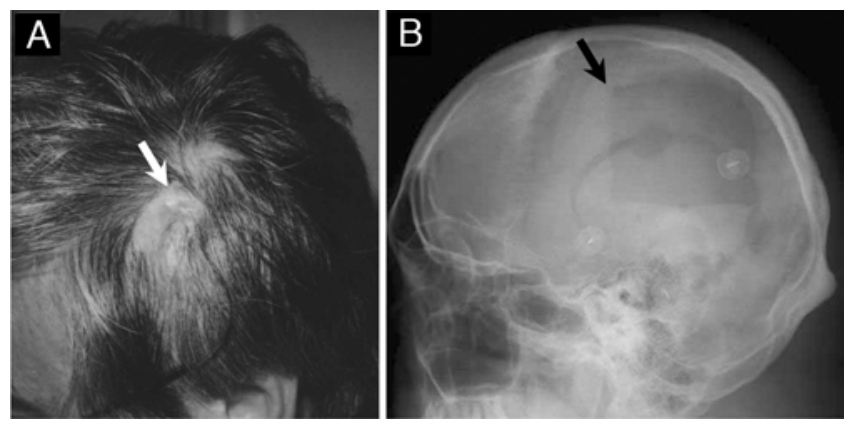

FIG. 2. Case 2. A: Preoperative photograph. The arrow indicates the fistula of the wound. B: Preoperative radiograph. The arrow indicates the same point indicated by the arrow in panel $\mathrm{A}$.
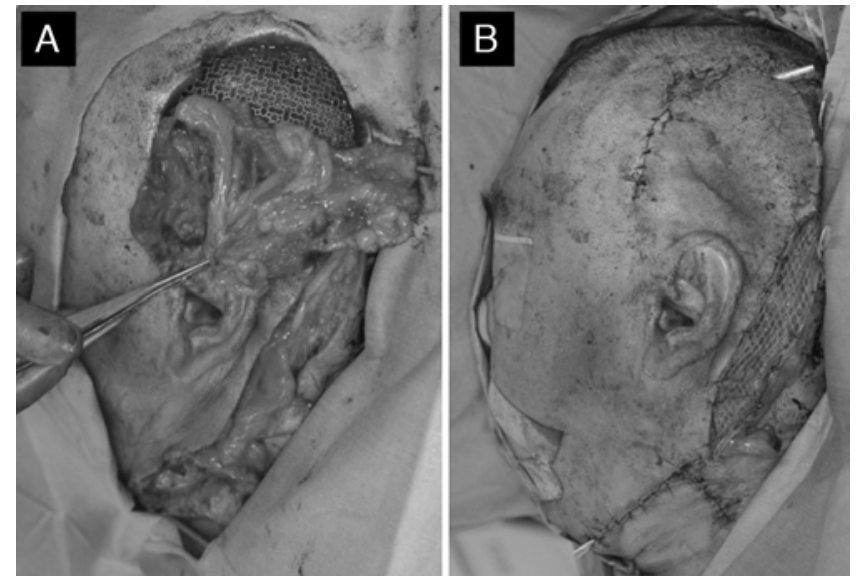

FIG. 3. Case 2. A: Intraoperative photograph. The omental flap was placed to enclose the titanium mesh. B: Postoperative photograph. A STSG was placed on the vascular pedicle of the omental flap.

on these reports, wound complications after craniotomy surgery without harvesting scalp vessels are estimated to occur in $10 \%-15 \%$ of all patients. However, once infection is involved, it develops into a serious problem. ${ }^{7}$ The infected bone should be removed and followed by a reconstructive procedure. Overall, operative wound complications are thought to occur at a higher incidence following intracranial revascularization surgeries than all craniotomies due to the repositioning of the vascular supply from the scalp to the brain. To date, there are only a few studies that focus on wound-related problems. Houkin et al. ${ }^{8}$ reported that wound complications that required plastic surgery, which is defined as a major complication in this report, were seen in 2 of 112 surgeries. Katsuta et al. ${ }^{10}$ claimed that cutaneous necrosis after revascularization surgery is strongly related to arteriosclerosis obliterans in the limbs and smoking, and may have a possible relation to the surgical procedure (flap method).

In our study, the overall wound complication rate was $21.4 \%$, which is higher than that in other reports. This may be because we counted minor wound problems, such as mere congestion or slight wound dehiscence, as wound complications. Minor wound complications appear to be relatively a high proportion (66.7\%) of all complications noted here. Most of these cases healed conservatively, but we think it is important to detect a complication and start
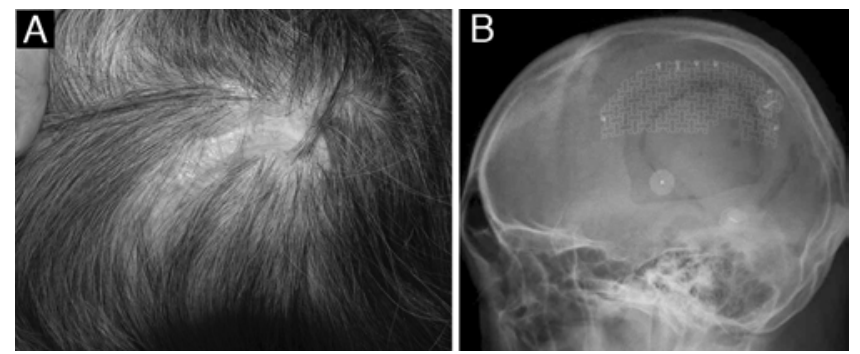

FIG. 4. Case 2. A: Postoperative photograph of the scalp. B: Postoperative radiograph. The bone defect was reconstructed using titanium mesh. 
treatment during the early phase. This leads to a better final outcome. Although a statistical difference in terms of the incidence of wound complications was not seen between the various surgical procedures, double-type procedures had significantly severer wound complications and a higher incidence of developing wound complications than single-type procedures $(\mathrm{p}=0.016$, by Cochran-Armitage trend test, Table 3; and $\mathrm{p}=0.048$ by multivariable logistic regression analysis, Table 4). Diabetes mellitus (DM) was also found to be a risk factor of wound complication. This makes sense in that microcirculation is intrinsically compromised in patients with $\mathrm{DM}$, and an invasive procedure may further worsen the condition. These results suggest that strict attention should be given to DM patients.

In contrast to the skin on other parts of the body, the scalp has a distinctive vascular anatomy where the galea aponeurotica, which corresponds to the muscular fascia layer in other bodily regions, carries the main blood supply to the scalp. Subcutaneous vascular communication is relatively poor because of the dense fibrous tissue that connects the subcutaneous and galeal layers. Because of this, the local flap of the scalp is usually elevated, including the galeal layer. Harvesting the blood vessels from the galeal layer decreases the scalp's blood supply. Even in procedures where the distal end of the vessel is continuously preserved rather than cut for anastomosis (e.g., EDAS and EDAMS), the blood supply of the galeal layer will be compromised as well. Thus, for surgeries that include blood vessel harvesting from the scalp, the skin incision, galeal incision, and vessel harvest line should be designed to compromise the blood supply as little as possible. Even for the simplest flap incision that places the harvested vessel in the middle of the flap (Fig. 5A), the cutaneous blood supply is considered to be halved. A cutaneous incision, galeal incision, and vessel harvest line that do not take this into account will result in skin necrosis (e.g., blood supply and drainage will be compromised in the area surrounded by the galeal incision and harvested vessel) (Fig. 5B). The linear curved incision is not considered to reduce blood supply during single-type procedures (Fig. 5C), which were frequently used until recently., ${ }^{3,10}$ In contrast, doubletype procedures, which we defined as those that harvest both the temporal and frontal branches of the STA, are considered to reduce the blood supply to the scalp in a critical way (Fig. 5D). The high wound complication rate in double-type procedures might largely stem from this reduction.

We believe other points that influence the postoperative wound complication rate include the following: traumatic versus atraumatic handling of the edge of tissue; strain to the suture line; extent of damage to the galeal blood vessels during vessel harvesting; and the size and location of the main vessels in the scalp. Considering these points may reduce the complication rate.

To create an effective treatment strategy, we classified wound complications into 2 groups. Minor wound complications included superficial congestion, superficial necrosis, and poor adaptation. These cases were conservatively treated with ointment or simple resuturing. Major wound complications included full-thickness skin necrosis with or without galeal layer necrosis. These were treated using a skin graft, local flap, or free flap. Treatment for major complications was decided based on the part and area of the necrotized tissue. Cranial bone infection was also classified as a major complication and treated by removing the infected bone flap, followed by reconstruction using either a free muscle flap or omental flap. ${ }^{2,9,15-19,24}$ When planning scalp reconstructive surgery after revascularization surgery, surgeons should consider some important points. The route of the vessel used in the primary surgery should be confirmed before surgery in order to avoid damage. The location and the size of the craniotomy and the area where the periosteum was damaged by the primary surgery should be clarified in order to understand which area must be covered with sturdy tissue. The reconstructive surgeon should consider these points and select the best procedure for successful wound coverage.
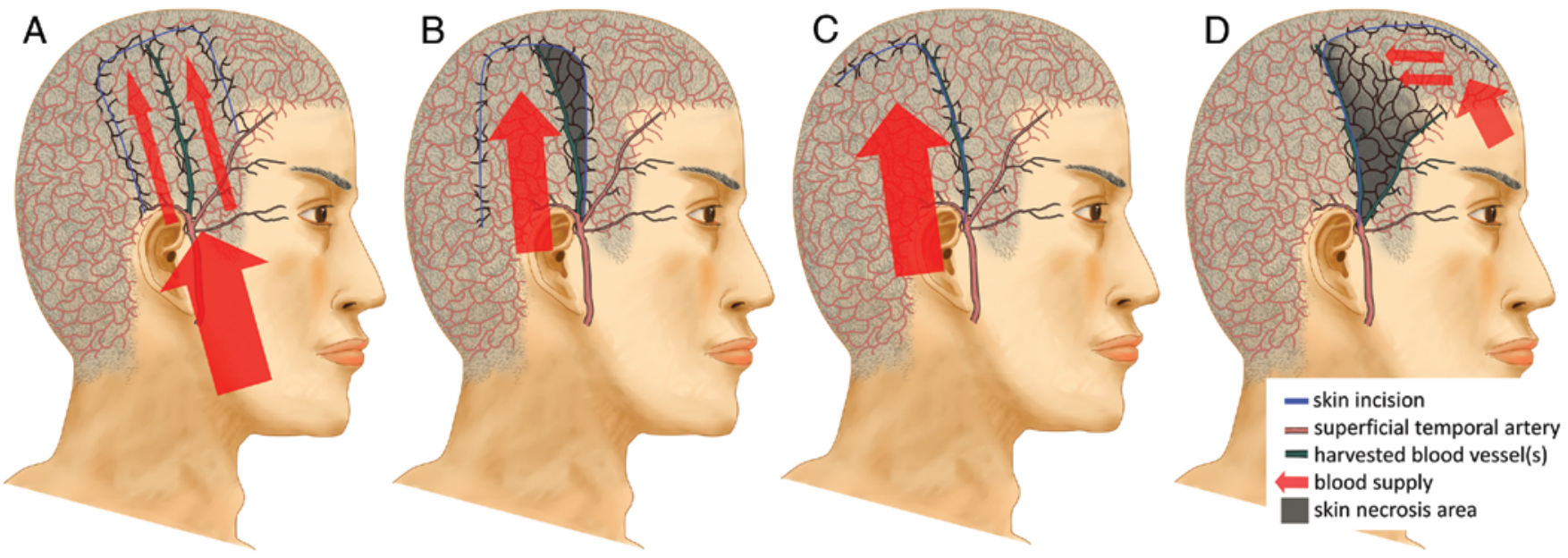

FIG. 5. A: Simple flap-type incision. The blood supply is halved by blood vessel harvesting. B: Flap incision does not consider the galeal blood supply. The area surrounded by the incision and harvested vessel will become either ischemic or congestive, which may result in skin necrosis. C: Linear curved line incision. The blood supply does not decrease. D: Double-type procedures critically decrease the blood supply. Copyright Keisuke Takanari. Published with permission. Figure is available in color online only. 


\section{Conclusions}

We reviewed the records of patients who underwent revascularization surgeries and retrospectively assessed the postoperative complications. Revascularization surgeries seemed to demonstrate a higher risk of wound-related complications. Double-type procedures, which use both branches of the STA, and a history of DM were found to be risk factors for wound-related complications. Attention should be paid to the design of the galeal incision and vessel harvest line. Also, special attention should be paid to patients with DM.

\section{References}

1. Abul-Hassan HS, von Drasek Ascher G, Acland RD: Surgical anatomy and blood supply of the fascial layers of the temporal region. Plast Reconstr Surg 77:17-28, 1986

2. Asai S, Kamei Y, Torii S: One-stage reconstruction of infected cranial defects using a titanium mesh plate enclosed in an omental flap. Ann Plast Surg 52:144-147, 2004

3. Aydin K, Cokluk C, Kuruoglu E, Gelmez S, Diren B, Rakunt $\mathrm{C}$, et al: Using the magnetic resonance three-dimensional volume rendering for tissues technique in the planning of craniotomy flaps with linear scalp incision. Minim Invasive Neurosurg 49:189-193, 2006

4. Baaj AA, Agazzi S, Sayed ZA, Toledo M, Spetzler RF, van Loveren H: Surgical management of moyamoya disease: a review. Neurosurg Focus 26(4):E7, 2009

5. Beheiry EE, Abdel-Hamid FA: An anatomical study of the temporal fascia and related temporal pads of fat. Plast Reconstr Surg 119:136-144, 2007

6. Dashti SR, Baharvahdat H, Spetzler RF, Sauvageau E, Chang SW, Stiefel MF, et al: Operative intracranial infection following craniotomy. Neurosurg Focus 24(6):E10, 2008

7. Delgado-López PD, Martín-Velasco V, Castilla-Díez JM, Galacho-Harriero AM, Rodríguez-Salazar A: Preservation of bone flap after craniotomy infection. Neurocirugia (Astur) 20:124-131, 2009

8. Houkin K, Ishikawa T, Yoshimoto T, Abe H: Direct and indirect revascularization for moyamoya disease surgical techniques and peri-operative complications. Clin Neurol Neurosurg 99 (Suppl 2):S142-S145, 1997

9. Iblher N, Ziegler MC, Penna V, Eisenhardt SU, Stark GB, Bannasch H: An algorithm for oncologic scalp reconstruction. Plast Reconstr Surg 126:450-459, 2010

10. Katsuta T, Inoue T, Arakawa S, Uda K: Cutaneous necrosis after superficial temporal artery-to-middle cerebral artery anastomosis: is it predictable or avoidable? Neurosurgery 49:879-884, 2001

11. Kawashima M, Rhoton AL Jr, Tanriover N, Ulm AJ, Yasuda A, Fujii K: Microsurgical anatomy of cerebral revascularization. Part I: anterior circulation. J Neurosurg 102:116-131, 2005

12. Kawashima M, Rhoton AL Jr, Tanriover N, Ulm AJ, Yasuda A, Fujii K: Microsurgical anatomy of cerebral revascularization. Part II: posterior circulation. J Neurosurg 102:132-147, 2005

13. Kleintjes WG: Forehead anatomy: arterial variations and venous link of the midline forehead flap. J Plast Reconstr Aesthet Surg 60:593-606, 2007
14. Kubo S, Takimoto H, Yoshimine T: Endoscopically assisted harvesting of the superficial temporal artery: technical note. Neurosurgery 52:982-985, 2003

15. Kwon H, Kim HJ, Yim YM, Jung SN: Reconstruction of scalp defect after Moyamoya disease surgery using an occipital pedicle V-Y advancement flap. J Craniofac Surg 19:1075-1079, 2008

16. Leedy JE, Janis JE, Rohrich RJ: Reconstruction of acquired scalp defects: an algorithmic approach. Plast Reconstr Surg 116:54e-72e, 2005

17. Mehrara BJ, Disa JJ, Pusic A: Scalp reconstruction. J Surg Oncol 94:504-508, 2006

18. Newman MI, Hanasono MM, Disa JJ, Cordeiro PG, Mehrara BJ: Scalp reconstruction: a 15-year experience. Ann Plast Surg 52:501-506, 2004

19. Oishi SN, Luce EA: The difficult scalp and skull wound. Clin Plast Surg 22:51-59, 1995

20. Origitano TC, Petruzzelli GJ, Leonetti JP, Vandevender D: Combined anterior and anterolateral approaches to the cranial base: complication analysis, avoidance, and management. Neurosurgery 58 (4 Suppl 2):ONS-327-ONS-337, 2006

21. Patel HC, McNamara IR, Al-Rawi PG, Kirkpatrick PJ: Improved cerebrovascular reactivity following low flow EC/IC bypass in patients with occlusive carotid disease. Br J Neurosurg 24:179-184, 2010

22. Pinar YA, Govsa F: Anatomy of the superficial temporal artery and its branches: its importance for surgery. Surg Radiol Anat 28:248-253, 2006

23. Seery GE: Surgical anatomy of the scalp. Dermatol Surg 28:581-587, 2002

24. Senyuva C, Yücel A, Okur I, Cansiz H, Sanus Z: Free rectus abdominis muscle flap for the treatment of complications after neurosurgical procedures. J Craniofac Surg 7:317-321, 1996

25. Smith SF, Simpson JM, Sekhon LH: What progress has been made in surgical management of patients with astrocytoma and oligodendroglioma in Australia over the last two decades? J Clin Neurosci 12:915-921, 2005

26. Stock AL, Collins HP, Davidson TM: Anatomy of the superficial temporal artery. Head Neck Surg 2:466-469, 1980

\section{Author Contributions}

Conception and design: Wakabayashi, Kamei. Acquisition of data: Araki, Okamoto, Yokoyama, Murotani, Matsui. Analysis and interpretation of data: Takanari, Araki, Okamoto, Sato, Yagi, Toriyama, Yokoyama, Murotani, Matsui, Kamei. Drafting the article: Takanari, Araki, Okamoto, Sato, Toriyama. Critically revising the article: Takanari, Araki, Wakabayashi. Reviewed submitted version of manuscript: all authors. Approved the final version of the manuscript on behalf of all authors: Takanari. Statistical analysis: Takanari. Administrative/technical/material support: Takanari.

\section{Correspondence}

Keisuke Takanari, Department of Plastic and Reconstructive Surgery, Nagoya University Graduate School of Medicine, 65 Tsurumai-cho Showa-ku, Nagoya, Aichi 464-8560, Japan. email: takanari@med.nagoya-u.ac.jp. 